\title{
The development of attenuation compensation models of fluorescence spectroscopy signals
}

\author{
Victor V. Dremin*a , Evgeny A. Zherebtsov ${ }^{\mathrm{a}}$, Ilya E. Rafailov ${ }^{\mathrm{b}}$, Andrey Y. Vinokurov ${ }^{\mathrm{c}}$, \\ Irina N. Novikova ${ }^{a}$, Angelina I. Zherebtsova ${ }^{a}$, Karina S. Litvinova ${ }^{b}$, Andrey V. Dunaev ${ }^{a}$ \\ ${ }^{a}$ Biomedical Photonics Instrumentation Group, Scientific-Educational Center "Biomedical \\ Engineering", State University - Education-Science-Production Complex, Oryol, 302020, Russia; \\ ${ }^{\mathrm{b}}$ School of Engineering and Applied Sciences, Aston Institute of Photonic Technologies, Aston \\ University, Aston Triangle, Birmingham, B4 7ET, UK; \\ "Scientific-Educational Center "Biotechnology and Chemical Technologies", State University - \\ Education-Science-Production Complex, Oryol, 302020, Russia; \\ 'Optoelectronics and Biomedical Photonics Group, Aston Institute of Photonic Technologies, Aston \\ University, Aston Triangle, Birmingham, B4 7ET, UK
}

\begin{abstract}
This study examines the effect of blood absorption on the endogenous fluorescence signal intensity of biological tissues. Experimental studies were conducted to identify these effects. To register the fluorescence intensity, the fluorescence spectroscopy method was employed. The intensity of the blood flow was measured by laser Doppler flowmetry.

We proposed one possible implementation of the Monte Carlo method for the theoretical analysis of the effect of blood on the fluorescence signals. The simulation is constructed as a four-layer skin optical model based on the known optical parameters of the skin with different levels of blood supply. With the help of the simulation, we demonstrate how the level of blood supply can affect the appearance of the fluorescence spectra.

In addition, to describe the properties of biological tissue, which may affect the fluorescence spectra, we turned to the method of diffuse reflectance spectroscopy (DRS). Using the spectral data provided by the DRS, the tissue attenuation effect can be extracted and used to correct the fluorescence spectra.
\end{abstract}

Keywords: fluorescence spectroscopy, laser Doppler flowmetry, Monte Carlo simulation, 3D model, diffuse reflectance spectroscopy, correction spectra.

\section{INTRODUCTION}

In recent years, fluorescence spectroscopy (FS) has been successfully used in studies of biological objects at the micro level, which allows the monitoring of temporal and spatial dynamics of molecular processes. This method is based on analysing the characteristics of induced endogenous fluorescence in probed biological tissues with low-intensity optical radiation at certain wavelengths ${ }^{1,2}$. However, in general, one of the main problems with this method so far, is the correct interpretation of the data ${ }^{3}$. It is known that changes in blood flow can affect the fluorescence spectra: it may result from signal attenuation due to the absorption capacity of the blood and the change in the concentration of fluorescent coenzyme occurring during concomitant changes in metabolism. In this study, we tried to experimentally study the effects of blood flow on the fluorescence signals in biological tissues and theoretically describe this effect.

Human skin (dermis) is rich in arterioles, venules and capillaries. Consequently, blood can introduce a unique component into the recorded fluorescence spectrum, absorbing the probing as well as the fluorescence radiation. Moreover, as is known ${ }^{4,5}$, depending on the percentage of oxyhemoglobin $\left(\mathrm{HbO}_{2}\right)$ in the total amount of hemoglobin, the blood has a different absorption spectra (fig.1).

*dremin_viktor@mail.ru; phone 74862 419876; bmecenter.ru/en/fluorescence_spectroscopy_in_vivo

Saratov Fall Meeting 2015: Third Annual Symposium Optics and Biophotonics; Seventh Finnish-Russian Photonics and Laser Symposium (PALS), edited by E. A. Genina, V. L. Derbov, D. E. Postnov, A. B. Pravdin, K. V. Larin, I. V. Meglinski, V. V. Tuchin, Proc. of SPIE Vol. 9917, 99170Y · (C) 2016 SPIE · CCC code: 1605-7422/16/\$18 · doi: 10.1117/12.2229451 


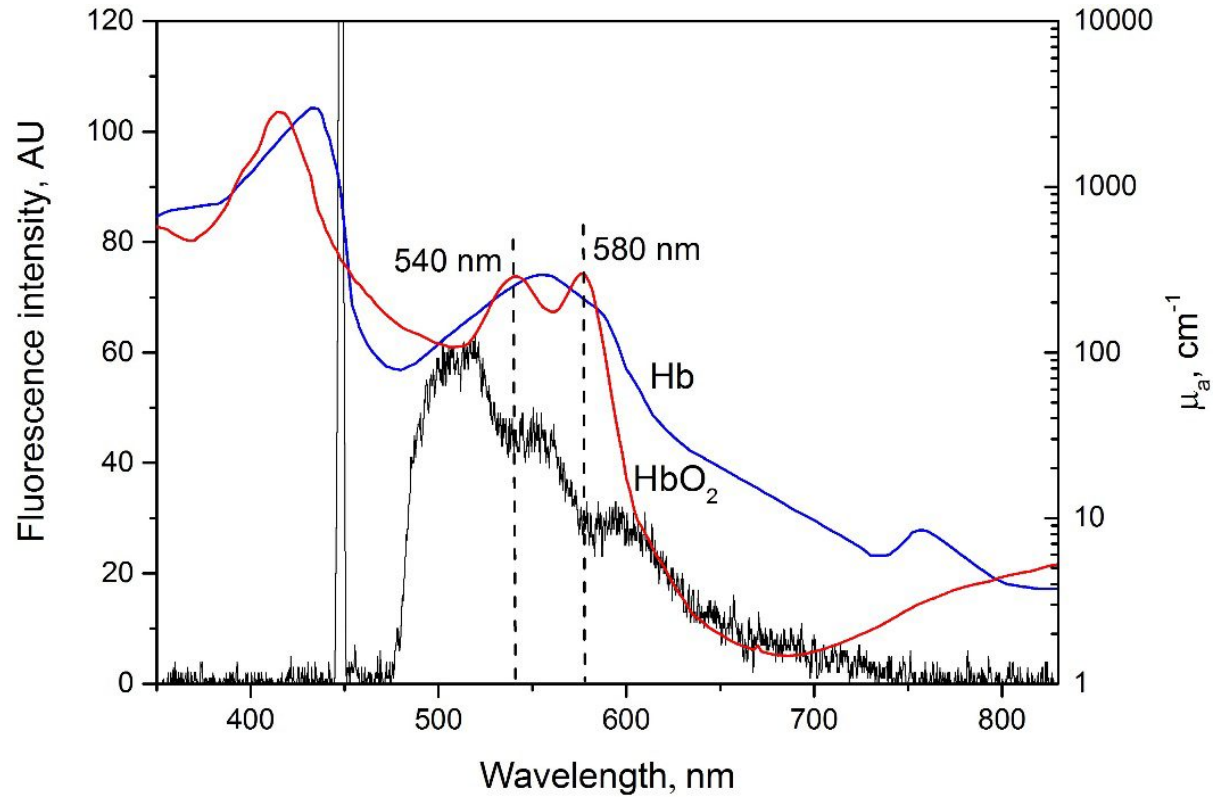

Figure 1. The absorption spectra of the different fractions of hemoglobin (oxy and deoxy-hemoglobin) and registered endogenous fluorescence spectrum of biological tissue on the volar surface of the distal phalanx of the the right hand middle finger upon $450 \mathrm{~nm}$ excitation.

As shown in fig.1, the level of blood supply to areas of research affects the fluorescence spectrum and does not allow to fully appreciate the content of any of the biomarker in the skin. Thus, the aim of this research was to experimentally study the effects of blood flow on the fluorescence signals in biological tissues and theoretically describe this effect.

\section{MATERIAL AND METHODS}

Experimental studies were conducted on the palmar surface of the skin of the middle and ring fingers of the right hand of healthy volunteers. To evaluate the intensity of blood flow, perfusion was recorded by laser Doppler flowmetry (LDF) with a "LAKK-02" laser analyser (sensing wavelength $-1064 \mathrm{~nm}$ ). The fluorescence spectrum of biological tissues was detected by means of non-invasive diagnostic FS channel complex "LAKK-M" (SPE "LAZMA", Russia) 6 for 2 excitation wavelengths of endogenous fluorophores NADH and FAD - 365 and $450 \mathrm{~nm}$, respectively. The main idea of the research was the implementation of provocative actions (functional tests) on blood flow through changes in extremity temperature (heat and cold pressor test) and application of the brachial arterial occlusion cuff with pressure of 200-220 $\mathrm{mm} \mathrm{Hg}$ (occlusion test - OT). Special tooling designed and printed on a 3D-printer, allows for the placement of the measuring LDF and FS channel fibres along the arm, as well as the ability to secure additional 2-channel temperature sensors specially developed for the temperature measuring instrument.

One study recorded the fluorescence of NADH or FAD. The study consisted of 7 stages, during which the fluorescence spectra and the perfusion of biological tissues are registered at the same time: background recording when placing your hands in the air $-2 \mathrm{~min}$; in warm water $\left(42^{\circ} \mathrm{C}\right)-4 \mathrm{~min}$ (heat test); in cold water $\left(15-20^{\circ} \mathrm{C}\right)-10 \mathrm{~min}$ (cold pressor test); occlusion of the brachial artery in cold water $-3 \mathrm{~min}$ (in conjunction with occlusive cold pressor test); relaxation step in cold water - $10 \mathrm{~min}$; heating of hot water $\left(42^{\circ} \mathrm{C}\right)-11 \mathrm{~min}$ (final thermal break). The duration of the experiment was a full 40 minutes, during which about 70-90 fluorescence spectra of biological tissues were recorded. A total of 37 experiments were performed on 10 volunteers. 


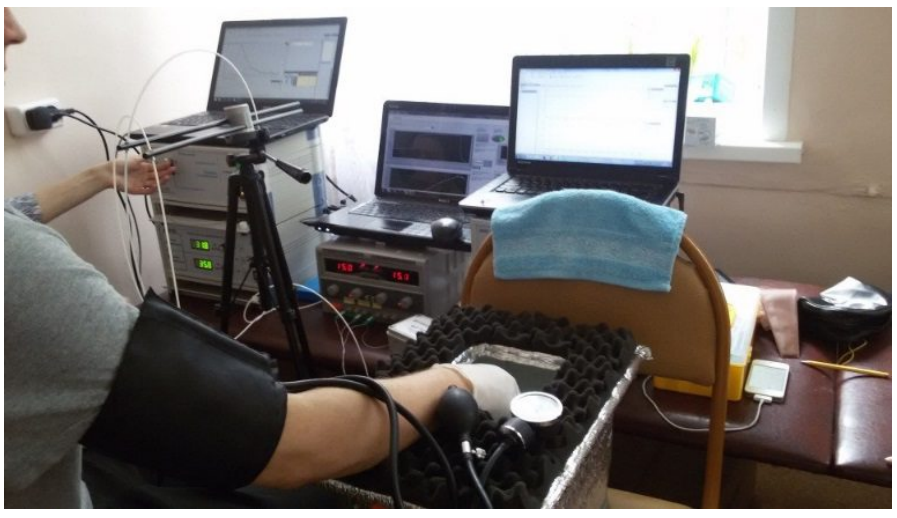

a

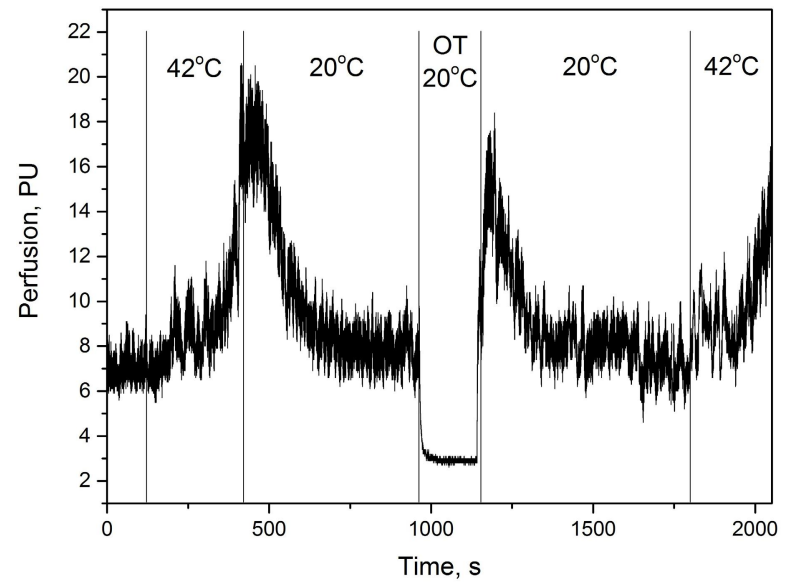

b

Figure 2. Experimental setup (a) including "LAKK-M" and "LAKK-02" systems and a timing chart of the experiment (b).

\section{EXPERIMENTAL RESULTS AND DISCUSSION}

With the help of non-invasive methods LDF and FS, we have shown the relationship between blood flow (perfusion) and recorded fluorescence signals.

For all volunteers, registered back reflected radiation and the intensity of fluorescence from biological tissue displayed a high inverse correlation ( 0.4-0.8) with perfusion and temperature for both fluorophores investigated. Thus, we can assume that factors affecting the absorption of the blood change the fluorescence spectra of biological tissue.

Mathematical models and methods of fluorescence spectra correction were examined. Creating analytical models is very difficult due to the complexity of solving the problem, even for simple cases. Numerical methods, including the probabilistic Monte Carlo, are often used to solve the problems of light propagation in biological tissue. In this work we propose one possible implementation of this method, as well as a simplified correction model based on Beer's law.

TracePro (Lambda Software) was used for the construction of the model. Principles of modelling inherent in the software have been described in detail in Rafailov et al $(2015)^{7}$.

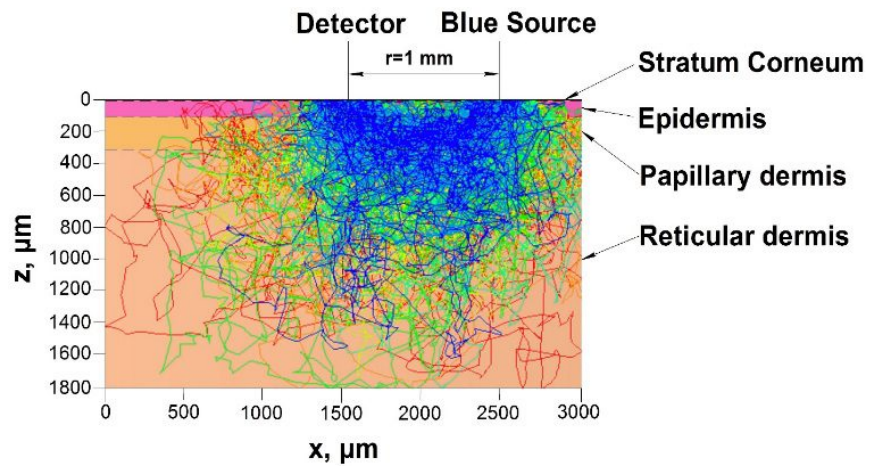

a

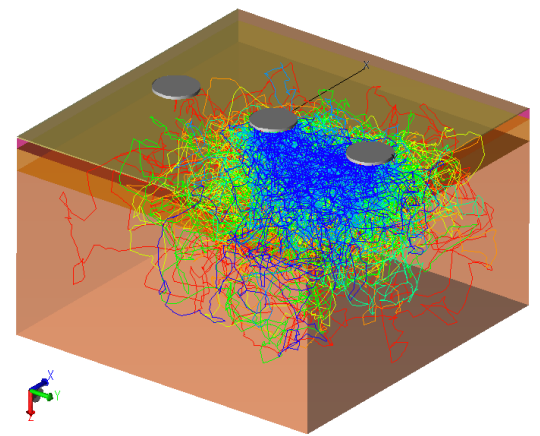

$\mathrm{b}$

Figure 3. Side view of 3D model (a), full 3D view of model (b). The lines indicate the passage of individual photons through the tissue. 
Figure 4 shows the potential of the proposed method for modeling the propagation of optical radiation through biological tissue. The calculation of transport parameters that determine the optical properties of biological tissues were conducted on the basis of the data presented in Jacques $(2013)^{5}$. The boundary conditions which are presented in Sinichkin et al $(1998)^{8}$ have been numerically simulated by the Monte Carlo method. Figure 4 shows how the content of the blood can affect the fluorescence spectrum of collagen, namely shifting its peak in longer wavelengths.

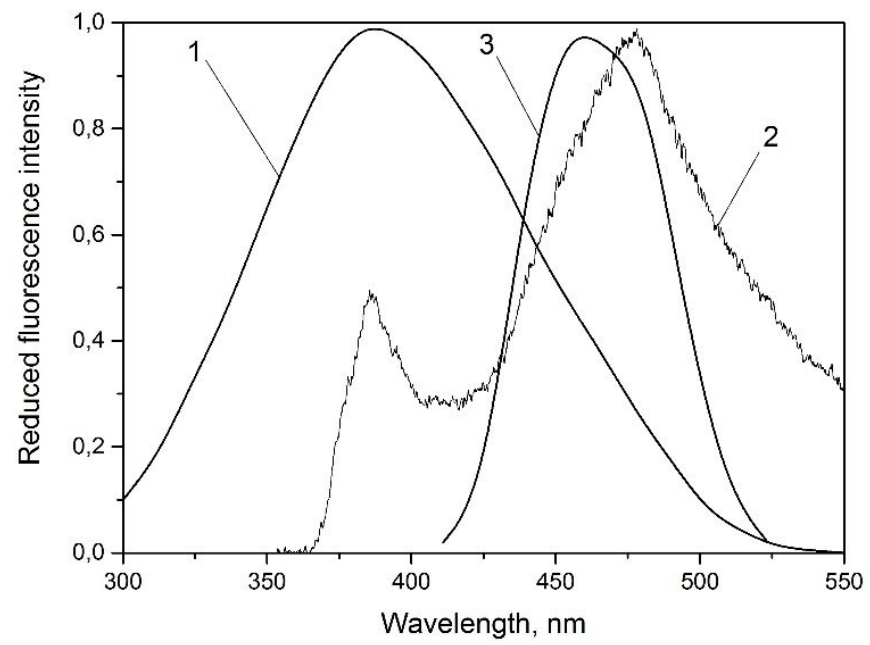

Figure 4. Experimental fluorescence spectra of pure collagen in vitro (1), of the skin in vivo (2) and modelled fluorescence spectrum of collagen with $5 \%$ content of the blood in the dermis (3).

Based on the developed optical model and further Monte Carlo simulations, the general distribution of light within tissue can be predicted. This can be invaluable in a variety of non-invasive optical methods of investigation, ultimately leading to potential new avenues of modelling and diagnostics.

To describe the properties of biological tissue, which may affect the fluorescence spectra, we turned to the method of diffuse reflectance spectroscopy (DRS). The method carries information about the morphology of the tissue, as well as the content of the different chromophores (including hemoglobin) ${ }^{9}$. Therefore using the spectral data provided by the DRS, tissue attenuation effect can be extracted and used to correct the fluorescence spectra. In many studies, the diffuse reflectance $R_{d}{ }^{10,11}$ or ${\sqrt{R_{d}}}^{12}$ may be directly used as an initial approximation of the correciton parameter which divides the fluorescence spectrum. In this work, we use an approximation of Beer's law to compute the light absorption coefficient of blood. An assumption can be made that the majority of photons undergo a very small number of scattering events before being collected by the probe. This fact satisfies the applicability of Beer's law in turbid media. The corrected fluorescence spectrum was calculated as

$$
F_{c o r}(\lambda)=\frac{F(\lambda)}{e^{\frac{l_{F}}{l_{R}} \ln (R(\lambda))}},
$$

where $F(\lambda)$ - the measured fluorescence signal; $R(\lambda)$ - the calculated diffuse reflectance signal; $l_{F}$ - pathlength of the fluorescence light; $l_{R}$ - pathlength of the diffuse reflectance light.

The reflection spectra is calculated using the formula: 


$$
R(\lambda)=\frac{R_{t}(\lambda)-R_{b}(\lambda)}{R_{P T F E}(\lambda)-R_{b}(\lambda)},
$$

where; $R_{t}(\lambda)$ - the measured diffuse reflectance signal of biological tissue; $R_{P T F E}(\lambda)$ - the measured diffuse reflectance signal of PTFE optical diffuser; $R_{b}(\lambda)$ - the background spectra obtained with the light source switched off.

As the source used white LED, it is integrated into the fiber of the "LAKK-M" complex. To register diffuse reflectance spectra spectrometric channel of complex "LAKK-M" was used.

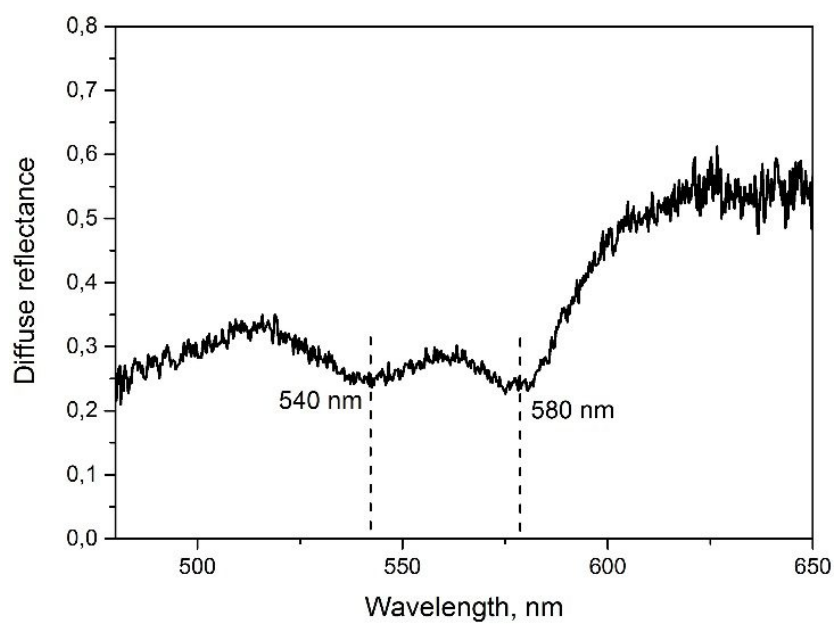

a)

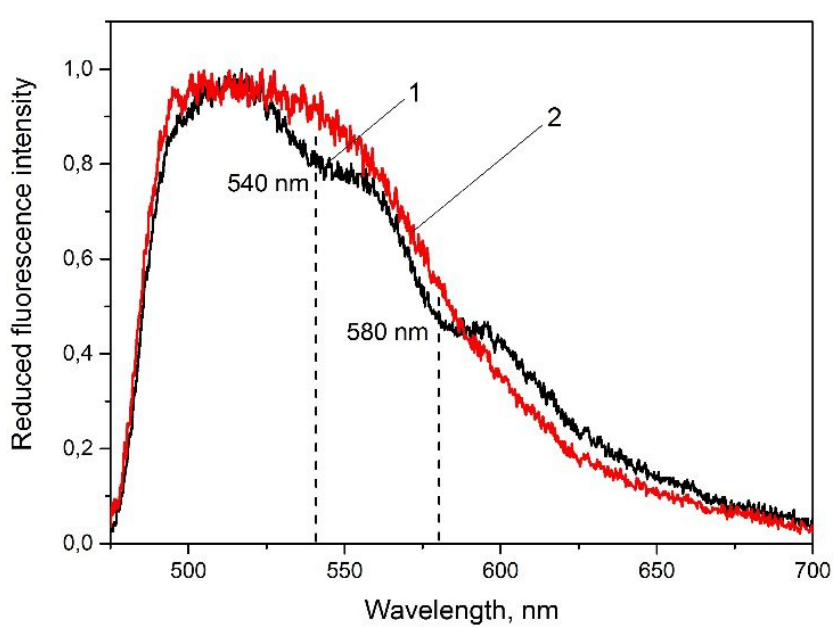

b)

Figure 5. Reflectance spectra (a); reduced registered (1) and corrected (2) fluorescence spectra (b) to biological tissue of volar surface of the of the middle finger distal phalanx of the right hand.

In formula (1) the ratio $\frac{l_{F}}{l_{R}}$ was evaluated by the criterion of least squares, minimizing function $\left[F_{O T}(\lambda)-F_{c o r}(\lambda)\right]^{2}$, where $F_{O T}(\lambda)$ - measured fluorescence signal during the occlusion test, in which the elimination of the influence of blood on the signal may be looked at. The fluorescence spectrum from a clear solution of the fluorophore of interest (without absorption or scattering) can be utilised as such a reference signal.

Figure $5 \mathrm{~b}$ shows a lack of absorption peaks of blood on the corrected spectra (red curve). Thus, it is possible to speak about the adequacy of the proposed methodology of spectra correction. From the data obtained, the combined use of several optical diagnostic methods is seen as one of the promising directions of development of this field of knowledge.

\section{CONCLUSION}

The results obtained must be considered in fluorescence spectroscopy research to determine the concentrations of various fluorophores in biological tissue and the development of new diagnostic criteria and normalizing factors. Also, the results of these experiments indicate a need for further studies, since this generally leads to improved methodology and instrumentation technology for use in medical fluorescence spectroscopy.

For the successful implementation of the methods of fluorescence spectroscopy in medical practice, it is important to search for simple and easily implemented methods for correction. The use of such methods for correction of the fluorescence spectra can be useful for research related to the change of blood flow, for example, in studying the metabolic activity of tissues in vivo, in various functional tests and others. 


\section{ACKNOWLEDGMENTS}

This work was supported by the grant of the Ministry of Education and Science, Russian Federation, for State University - Education-Science-Production Complex (state task, GZ №310).

\section{REFERENCES}

[1] Lakowicz, J. R., [Principles of Fluorescence Spectroscopy], Kluwer Academic Publishers, New York, 954 (2006).

[2] Mycek, M. A. and Pogue, B. W., [Handbook of Biomedical Fluorescence], Taylor \& Francis, (2003).

[3] Dunaev, A. V., Dremin, V. V., Zherebtsov, E. A., Rafailov, I. E., Litvinova, K. S., Palmer, S. G., Stewart, N. A., Sokolovski, S. G. and Rafailov, E. U., "Individual variability analysis of fluorescence parameters measured in skin with different levels of nutritive blood flow," Med Eng Phys, 37(6), 574-83 (2015).

[4] Jacques, S. L., [Skin Optics], http://omlc.org/news/jan98/skinoptics.html (1998).

[5] Jacques, S. L., "Optical properties of biological tissues: a review," Phys Med Biol, 58(11), R37-61 (2013).

[6] Rogatkin, D. A., Sokolovski, S. G., Fedorova, K. A., Sidorov, V. V., Stewart, N. A. and Rafailov, E. U., "Basic principles of design and functioning of multifunctional laser diagnostic system for non-invasive medical spectrophotometry," Proc. SPIE. 7890, 78901H1 (2011).

[7] Rafailov, I., Palmer, S., Litvinova, K., Dremin, V., Dunaev, A. and Nabi, G., "A novel excitation-emission wavelength model to facilitate the diagnosis of urinary bladder diseases," Proc. SPIE. 9303, 93030W (2015).

[8] Sinichkin, Y. P., Utz, S. R., Mavlutov, A. H. and Pilipenko, H. A., "In vivo fluorescence spectroscopy of the human skin: experiments and models," Journal of Biomedical Optics, 3, 201-211 (1998).

[9] Anderson, R. R. and Parrish, J. A., "The optics of human skin,” J Invest Dermatol, 77(1), 13-9 (1981).

[10] Zeng, H., MacAulay, C., McLean, D. I. and Palcic, B., "Spectroscopic and microscopic characteristics of human skin autofluorescence emission," Photochemistry and Photobiology, 61(6), 639-645 (1995).

[11] Qu, J. Y. and Hua, J., "Calibrated fluorescence imaging of tissue in vivo," Applied Physics Letters, 78(25), 4040-4042 (2001).

[12] Utz, S. R., Knuschke, P. and Sinichkin, Y. P., "In vivo evalution of sunscreens by spectroscopic methods," Skin Research and Technology, 2(3), 114-121 (1996). 